\title{
THE WAVE PROCESSES IN THREE-LAYER SHELLS OF ROTATIONWITH TAKING INTO ACCOUNT THE DISCRETE FILLER AT NON-STATIONARY LOADS
}

\section{ВОЛНОВЫЕ ПРОЦЕССЫ В ТРЕХСЛОЙНЫХ ОБОЛОЧКАХ ВРАЩЕНИЯ С УЧЕТОМ ДИСКРЕТНОСТИ ЗАПОЛНИТЕЛЯ ПРИ НЕСТАЦИОНАРНЫХ НАГРУЗКАХ}

\author{
Vladimyr Meish ${ }^{1}$ \\ Yuliia Meish $^{2}$
}

DOI: https://doi.org/10.30525/978-9934-26-021-6-36

Abstract. Thin-walled shell structures in the form of plates and shells of various shapes have a high bearing capacity, lightness, and relative ease of manufacture. Three-layer shell elements, which consist of two bearing layers and a filler, which ensures their joint work, are widely used in mechanical engineering, industrial and civil construction, aviation and space technology, shipbuilding. When calculating the strength of three-layer shell structures with a discrete filler under dynamic loads, it becomes necessary to determine the stress-strain state both in the area of a sharp change in the geometry of the structure and at a considerable distance from the heterogeneity. The complexity of the processes that arise in this case necessitates the use of modern numerical methods for solving dynamic problems of the behavior of three-layer shell elements with a discrete filler. In this regard, the determination of the stress-strain state of three-layer shells with a discrete filler under nonstationary loads and the development of an effective numerical method for solving problems of this class is an urgent problem in the mechanics of a deformable solid. On the basis of the theory of threelayered shells with applying the hypotheses for each layer the nonstationary vibrations threelayered shells of revolution with allowance of discrete fillers are

\footnotetext{
${ }^{1}$ Doctor of Science (Physics and Mathematics),

Professor, Main researcher, S.P. Timoshenko Institute of Mechanics of the National Academy of Sciences of Ukraine, Ukraine

${ }^{2}$ Doctor of Science (Technology), Associate Professor,

Professor of the Higher Mathematics Department,

National Transport University, Ukraine

(C) Vladimyr Meish, Yuliia Meish
} 
investigated. Hamilton-Ostrogradskyy variational principle for dynamical processes is used for deduction of the motion equations. An efficient numerical method for solution of problems on nonstationary behaviour of threelayers shells of revolution with allowance of discrete fillers are used. The wide diapason of geometrical, and physico-mechanical parameters of nonhomohenes threelayered structure are considerated. On the basis of the offered model nonstationary problems of the forced nonlinear vibrations of threelayered shells of revolution of various structure are solved and analysed. The basis of the developed numerical method for the study of nonstationary oscillations is the application of explicit finite-difference schemes to solve the initial differential equations in partial derivatives. The theory is based on the relations of the theory of three-layer shells of revolution taking into account the discreteness of the filler, which are based on the hypotheses of the geometrically nonlinear theory of shells and rods of the Timoshenko type.

\section{1. Введение}

Тонкостенные неоднородные элементы конструкций в виде пластинок и оболочек различной формы находят широкое применение в машиностроении, промышленном и гражданском строительстве, авиационной и космической технике, судостроении. Сюда можно отнести подкрепленные оболочки, трехслойные оболочки и т. д. [2; 8-12]. Широкое распространение получили трехслойные оболочечные элементы, которые состоят из двух несущих слоев и заполнителя, который обеспечивает их совместную работу. В ряде случаев заполнитель имеет дискретную структуру, что затрудняет расчет указанных оболочечных элементов, в частности, при нестационарных нагрузках [8]. При расчете на прочность трехслойных оболочечных структур с дискретным заполнителем при динамических нагрузках возникает необходимость определить напряженно-деформированное состояние как в области резкого изменения геометрии конструкции, так и на значительном расстоянии от неоднородности. При этом, одной из особенностей исследуемого процесса является волновая природа и распределение полей физико-механических параметров во всей системе. Сложность процессов, которые при этом возникают, обусловливает необходимость применения современных численных методов решения динамических задач поведения трехслойных оболочечных элементов с дискретным 
заполнителем. В связи с этим, определение напряженно-деформированного состояния трехслойных оболочек с дискретным заполнителем при нестационарных нагрузках и развитие эффективного численного метода решения задач данного класса представляет собой актуальную задачу механики деформируемого твердого тела. В основу построения теории положены соотношения теории трехслойных оболочек вращения с учетом дискретности заполнителя, которые основываются на гипотезах геометрически нелинейной теории оболочек и стержней типа Тимошенко [2]. При разработке численной методики исследования нестационарных колебаний лежит применение явных конечно-разностных схем к решению исходных дифференциальных уравнений в частных производных [2; 4; 7].

\section{2. Постановка задачи}

Трехслойная оболочка с дискретным внутренним заполнителем представляет собой упругую структуру, которая состоит из внутренней (индекс 1) и внешней (индекс 2) обшивок и набора дискретных ребер, жестко соединенных с указанными обшивками. В частности, это может быть оболочка с дискретным набором шпангоутов, или оболочка с продольным дискретным набором ребер, или трехслойная оболочка с продольно-поперечным дискретным ребристым заполнителем, схематическое изображение элемента конструкции представлено на рис. 1.

Принимается, что напряженно-деформированное состояние обшивок может быть определено в рамках геометрически нелинейной теории оболочек типа Тимошенко [2]. Для расчета дискретных ребер принимается геометрически нелинейной вариант теории стержней Тимошенко [2].

Деформированное состояние внутренней и внешней обшивок может быть определено через компоненты обобщенного вектора перемещений $\bar{U}_{1}=\left(u_{1}^{1}, u_{2}^{1}, u_{3}^{1}, \phi_{1}^{1}, \phi_{2}^{1}\right)^{T}$ и $\bar{U}_{2}=\left(u_{1}^{2}, u_{2}^{2}, u_{3}^{2}, \phi_{1}^{2}, \phi_{2}^{2}\right)^{T}$. Деформированное состояние ребра, направленного вдоль оси $\alpha_{1}$, будем определять обобщенным вектором перемещений $\bar{U}_{i}=\left(u_{1 i}, u_{2 i}, u_{3 i}, \phi_{1 i}, \phi_{2 i}\right)^{T}$. Соответственно, ребра, направленного вдоль оси $\alpha_{2}-$ вектором $\bar{U}_{j}=\left(u_{1 j}, u_{2 j}, u_{3 j}, \phi_{1 j}, \phi_{2 j}\right)^{T}$.

Для вывода уравнений колебаний трехслойной упругой структуры с дискретным заполнителем используется вариационный принцип стационарности Гамильтона-Остроградского [2], согласно которому 


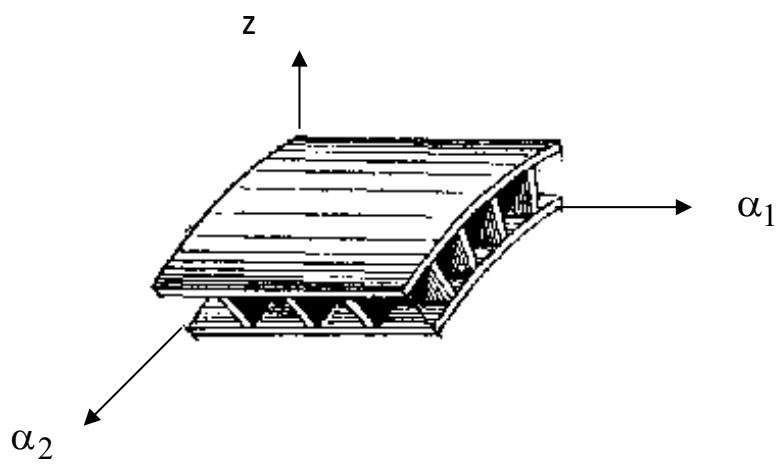

Рис. 1

$$
\delta \int_{t_{1}}^{t_{2}}(\mathrm{~K}-\Pi+\mathrm{A}) d t=0,
$$

где $\mathrm{K}$ - полная кинетическая энергия упругой системы, П - полная потенциальная энергия упругой системы, А-работа внешних сил, $t_{1}$ и $t_{2}$ - фиксированные моменты времени.

Записывая выражения для потенциальной и кинетической энергий [2] для оболочки и ребер и выполняя операцию варьирования в выражении (2.1) после стандартных преобразований получим четыре системы уравнений:

- уравнения колебаний внутренней и внешней гладких оболочек (соответственно индексы 1 и 2)

$$
\begin{gathered}
\frac{1}{A_{1}^{i} A_{2}^{i}}\left[\frac{\partial}{\partial \alpha_{1}}\left(A_{2}^{i} T_{11}^{i}\right)-\frac{\partial A_{2}^{i}}{\partial \alpha_{1}} T_{22}^{i}+\right. \\
\left.+\frac{\partial}{\partial \alpha_{2}} A_{1}^{i}\left(S^{i}+k_{1} H^{i}\right)+\frac{\partial A_{1}^{i}}{\partial \alpha_{1}}\left(S^{i}+k_{2} H^{i}\right)\right]+k_{1}^{i} \bar{T}_{13}^{i}+P_{1}^{i}=\rho_{i} h_{i} \frac{\partial^{2} u_{1}^{i}}{\partial t^{2}} \\
\frac{1}{A_{1}^{i} A_{2}^{i}}\left[\frac{\partial}{\partial \alpha_{2}}\left(A_{1}^{i} T_{22}^{i}\right)-\frac{\partial A_{1}^{i}}{\partial \alpha_{2}} T_{11}^{i}+\frac{\partial}{\partial \alpha_{1}} A_{2}^{i}\left(S^{i}+k_{2} H^{i}\right)+\frac{\partial A_{2}^{i}}{\partial \alpha_{1}}\left(S^{i}+k_{1} H^{i}\right)\right]+ \\
+k_{2}^{i} \bar{T}_{23}^{i}+P_{2}^{i}=\rho_{i} h_{i} \frac{\partial^{2} u_{2}^{i}}{\partial t^{2}},
\end{gathered}
$$




$$
\begin{aligned}
& \frac{1}{A_{1}^{i} A_{2}^{i}}\left[\frac{\partial}{\partial \alpha_{1}}\left(A_{2}^{i} \bar{T}_{13}^{i}\right)+\frac{\partial}{\partial \alpha_{2}}\left(A_{2}^{i} \bar{T}_{23}^{i}\right)\right]-k_{2}^{i} T_{22}^{i}+P_{3}^{i}=\rho_{i} h_{i} \frac{\partial^{2} u_{3}^{i}}{\partial t^{2}} \\
& \frac{1}{A_{1}^{i} A_{2}^{i}}\left[\frac{\partial}{\partial \alpha_{1}}\left(A_{2}^{i} M_{11}^{i}\right)-\frac{\partial A_{2}^{i}}{\partial \alpha_{1}} M_{22}^{i}+\right. \\
& \left.+\frac{\partial}{\partial \alpha_{2}}\left(A_{1}^{i} H^{i}\right)+\frac{\partial A_{1}^{i}}{\partial \alpha_{2}} H^{i}\right]-T_{13}^{i}=\rho_{i} \frac{h_{i}^{3}}{12} \frac{\partial^{2} \phi_{1}}{\partial t^{2}}, \\
& \frac{1}{A_{1}^{i} A_{2}^{i}}\left[\frac{\partial}{\partial \alpha_{1}}\left(A_{1}^{i} M_{22}^{i}\right)-\frac{\partial A_{1}^{i}}{\partial \alpha_{2}} M_{11}^{i}+\frac{\partial}{\partial \alpha_{1}}\left(A_{2}^{i} H^{i}\right)+\frac{\partial A_{2}^{i}}{\partial \alpha_{1}} H^{i}\right]-T_{23}^{i}=\rho_{i} \frac{h_{i}^{3}}{12} \frac{\partial^{2} \phi_{2}}{\partial t^{2}}, \\
& \bar{T}_{13}^{i}=T_{13}^{i}+T_{11}^{i} \theta_{1}^{i}+S^{i} \theta_{2}^{i}, \bar{T}_{23}^{i}=T_{23}^{i}+T_{22}^{i} \theta_{2}^{i}+S^{i} \theta_{1}^{i}, \\
& S^{i}=T_{12}^{i}-M_{21}^{i} / R_{2 i}, \quad H^{i}=M_{12}^{i}=M_{21}^{i} ;
\end{aligned}
$$

- уравнения колебаний і-го дискретного ребра заполнителя, направленного вдоль оси $\alpha_{1}$

$$
\begin{gathered}
{[\tilde{S}]_{i}+\frac{1}{A_{1}} \frac{\partial T_{11 i}}{\partial \alpha_{1}}+k_{1} \bar{T}_{13 i}=\rho_{i} F_{i} \frac{\partial^{2} u_{1 i}}{\partial t^{2}},} \\
{\left[T_{22}\right]_{i}+\frac{1}{A_{1}} \frac{\partial \bar{T}_{12 i}}{\partial \alpha_{1}}=\rho_{i} F_{i} \frac{\partial^{2} u_{2 i}}{\partial t^{2}},\left[\bar{T}_{23}\right]_{i}+\frac{1}{A_{1}} \frac{\partial \bar{T}_{13 i}}{\partial \alpha_{1}}-T_{11 i} k_{1 i}=\rho_{i} F_{i} \frac{\partial^{2} u_{3 i}}{\partial t^{2}},} \\
{[H]_{i}+\frac{1}{A_{1}} \frac{\partial M_{11 i}}{\partial \alpha_{1}}-T_{13 i}=\rho_{i} I_{1 i} \frac{\partial^{2} \phi_{1 i}}{\partial t^{2}},\left[M_{22}\right]_{i}+\frac{1}{A_{1}} \frac{\partial M_{12 i}}{\partial \alpha_{1}}=\rho_{i} I_{k r i} \frac{\partial^{2} \phi_{2 i}}{\partial t^{2}} ;}
\end{gathered}
$$

- уравнение колебаний ј-го дискретного ребра заполнителя, направленного вдоль $\alpha_{2}$

$$
\begin{array}{r}
{\left[T_{11}\right]_{j}+\frac{1}{A_{2}} \frac{\partial \bar{T}_{21 j}}{\partial \alpha_{2}}=\rho_{j} F_{j} \frac{\partial^{2} u_{1 j}}{\partial t^{2}},} \\
{[\tilde{S}]_{j}+\frac{1}{A_{2}} \frac{\partial T_{22 j}}{\partial \alpha_{2}}+k_{2 j} \bar{T}_{23 j}=\rho_{j} F_{j} \frac{\partial^{2} u_{2 j}}{\partial t^{2}},} \\
{\left[\bar{T}_{13 j}\right]_{j}+\frac{1}{A_{2}} \frac{\partial \bar{T}_{13 j}}{\partial \alpha_{2}}-k_{2 j} T_{22 j}=\rho_{j} F_{j} \frac{\partial^{2} u_{3 j}}{\partial t^{2}},}
\end{array}
$$




$$
\begin{gathered}
{\left[M_{11}\right]_{j}+\frac{1}{A_{2}} \frac{\partial M_{21 j}}{\partial \alpha_{2}}=\rho_{j} I_{k r j} \frac{\partial^{2} \phi_{1 j}}{\partial t^{2}},} \\
{[H]_{j}+\frac{1}{A_{2}} \frac{\partial M_{22 j}}{\partial \alpha_{2}}-T_{23}=\rho_{j} I_{2 j} \frac{\partial^{2} \phi_{2 j}}{\partial t^{2}} .}
\end{gathered}
$$

В соотношениях (2.3), (2.4) обозначения типа $[S]_{i}$ соответствуют суммарному действию величин усилий-моментов внутренней и внешней обшивок на i-й (или j-й) дискретный элемент заполнителя.

Связь между компонентами векторов перемещений, описывающих напряженно-деформированное состояние обшивок, и соответствующими величинами дискретных подкрепляющих элементов записываются согласно формул

$$
\begin{gathered}
u_{1 i}\left(\alpha_{1}\right)=u_{1}^{k}\left(\alpha_{1}, \alpha_{2 i}\right) \pm H_{i}^{k} \phi_{1}^{k}\left(\alpha_{1}, \alpha_{2 i}\right), \\
u_{2 i}\left(\alpha_{1}\right)=u_{2}^{k}\left(\alpha_{1}, \alpha_{2 i}\right) \pm H_{i}^{k} \phi_{2}^{k}\left(\alpha_{1}, \alpha_{2 i}\right), \\
u_{3 i}\left(\alpha_{1}\right)=u_{3}^{k}\left(\alpha_{1}, \alpha_{2 i}\right), \phi_{1 i}\left(\alpha_{1}\right)=\phi_{1}^{k}\left(\alpha_{1}, \alpha_{2 i}\right), \\
\phi_{2 i}\left(\alpha_{1}\right)=\phi_{2}^{k}\left(\alpha_{1}, \alpha_{2 i}\right), \quad k=1,2 ;
\end{gathered}
$$

- для обшивок и дискретных поперечных ребер заполнителя

$$
\begin{gathered}
u_{1 j}\left(\alpha_{2}\right)=u_{2}^{k}\left(\alpha_{1 j}, \alpha_{2}\right) \pm H_{j}^{k} \phi_{2}^{k}\left(\alpha_{1 j}, \alpha_{2}\right), \\
u_{2 j}\left(\alpha_{2}\right)=u_{1}^{k}\left(\alpha_{1 j}, \alpha_{2}\right) \pm H_{j}^{k} \phi_{1}^{k}\left(\alpha_{1 j}, \alpha_{2}\right), \\
u_{3 j}\left(\alpha_{2}\right)=u_{3}^{k}\left(\alpha_{1 j}, \alpha_{2}\right), \phi_{1 j}\left(\alpha_{2}\right)=\phi_{2}^{k}\left(\alpha_{1 j}, \alpha_{2}\right), \\
\phi_{2 j}\left(\alpha_{2}\right)=\phi_{1}^{k}\left(\alpha_{1 j}, \alpha_{2}\right), \quad k=1,2 .
\end{gathered}
$$

Обозначения в уравнениях (2.2) - (2.6) приняты согласно [1-4]. Уравнения движения неоднородной упругой структуры дополняются соответствующих граничными и начальными условиями, которые вытекают из вариационного принципа (2.1).

\section{3. Численный алгоритм решения задачи}

В случае осесимметричных колебаний трехслойных оболочек вращения с дискретным заполнителем, уравнения колебаний записываются в виде (2.2) - (2.6). Рассматривается пространственный интервал $s_{0} \leq s \leq s_{N}, s=A_{1} \alpha_{1}$. Производится разбиение интервала на $\mathrm{N}$ равных частей с дискретным шагом $\Delta s=s / N$. Вводится разностная 
сетка в полуцелый узлах. Компоненты обобщенных векторов перемещений обшивок и ребер сносим в целые узлы разностной сетки $u_{1}^{k}, u_{3}^{k}, \phi_{1}^{k} \rightarrow\left(u_{1}^{k}\right)_{l}^{n}, \quad\left(u_{3}^{k}\right)_{l}^{n}, \quad\left(\phi_{1}^{k}\right)_{l}^{n}, \quad k=1,2$;

$$
u_{1 j}, u_{3 j}, \phi_{1 j} \rightarrow\left(u_{1 j}\right)_{l}^{n},\left(u_{3 j}\right)_{l}^{n},\left(u_{1 j}\right)_{l}^{n}, \quad j=\overline{1, J} .
$$

Построение разностной схемы проводится с использованием интегро-интерполяционного метода построения конечно-разностных схем $[2 ; 7]$. Рассматриваются уравнения колебаний (2.2)-(2.6) в области

$$
\left\{s_{l-1 / 2} \leq s \leq s_{l+1 / 2}, t_{n-1 / 2} \leq t \leq t_{n+1 / 2}\right\} .
$$

Проводится интегрирования левых и правых частей уравнений по соответствующим переменным рассматриваемой области. В матрично-векторном виде разностные уравнения можно представить следующим образом

$$
[C] \bar{U}+[M] \frac{\partial^{2} \bar{U}}{\partial t^{2}}=\bar{F}(t),
$$

где $[\mathrm{M}]$ и $[\mathrm{C}]-$ матрицы массы жесткости дискретной разностной системы, $\bar{U}$ и $\bar{F}$ - векторы дискретных перемещений и внешней импульсной нагрузки.

Считая, что матрица [M] невирожденная, запишем последнее уравнение в виде

$$
[D] \bar{U}+\frac{\partial^{2} \bar{U}}{\partial t^{2}}=[M]^{-1} \bar{F}(t)
$$

где матрица $[D]=[M]^{-1}[C]$.

В работе [4] показано, что при использовании явной конечно-разностной схемы для интегрирования уравнений необходимым условием устойчивости разностных уравнений является условие вида

$$
\tau \leq 2 / \Omega_{\max }=2 \sqrt{\beta(D)},
$$

где $\Omega_{\text {max }}$ - максимальная частота собственных колебаний разностной системы; $\beta(D)$ - верхний предел спектра матрицы [D].

\section{4. Численные результаты}

Цилиндрическая оболочка. В случае трехслойной цилиндрической оболочки с конструктивно-ортотропным заполнителем уравнения колебаний имеют вид 


$$
\begin{gathered}
\sum_{i=1}^{2} L_{1}^{i}\left(\bar{U}_{i}\right)=\left(\sum_{i=1}^{2} \rho_{i} h_{i}+\rho_{z} \frac{F_{z}}{a}\right) \frac{\partial^{2} u_{1}}{\partial t^{2}}, \\
\sum_{i=1}^{2} L_{2}^{i}\left(\bar{U}_{i}\right)-\frac{T_{22 i}}{R_{z}}+P_{3}=\left(\sum_{i=1}^{2} \rho_{i} h_{i}+\rho_{z} \frac{F_{z}}{a}\right) \frac{\partial^{2} u_{3}}{\partial t^{2}}, \\
\sum_{i=1}^{2} L_{3}^{i}\left(\bar{U}_{i}\right)=\left(\sum_{i=1}^{2} \rho_{i} \frac{h_{i}^{3}}{12}+\rho_{z} \frac{I_{z}}{a}\right) \frac{\partial^{2} \phi_{1}}{\partial t^{2}}
\end{gathered}
$$

где

$$
\begin{aligned}
L_{1}^{i}\left(\bar{U}_{i}\right) & =\frac{\partial T_{11}^{i}}{\partial x}, \quad L_{2}^{i}\left(\bar{U}_{i}\right)=\frac{\partial \bar{T}_{13}^{i}}{\partial x}-\frac{T_{22}^{i}}{R_{i}}, \\
L_{3}^{i}\left(\bar{U}_{i}\right) & =\frac{\partial M_{11}^{i}}{\partial x}-T_{13}^{i}, \bar{T}_{13}^{i}=T_{13}^{i}+T_{11}^{i} \theta_{1} .
\end{aligned}
$$

Уравнения движения (4.1) дополняются соответствующими начальными и граничными условиями. Для случая уравнений колебаний с учетом дискретности заполнителя для жестко защемленных краев граничные условия полагались при $x=0, x=L(\mathrm{~L}-$ длина оболочки) в виде $u_{1}^{k}=u_{3}^{k}=\phi_{1}^{k}=0, k=1,2$. Принимались нулевые начальные условия.

Для случая уравнений колебаний с конструктивно-ортотропным заполнителем для жестко защемленных краев граничные условия полагались при $x=0, x=L$ в виде: $u_{1}=u_{3}=\phi_{1}=0$. Начальные условия нулевые.

Задача колебаний трехслойной цилиндрической оболочки согласно указанных теорий рассматривалась при следующих геометрических и физико-механических параметрах $L / h_{1}=40 ; h_{1}=h_{2} ; R_{1} / h_{1}=10 ; H_{j} / h_{1}=2 ; F_{j}=H_{j} h_{1} ; E_{1}^{1}=E_{1}^{2}=E_{j}=7 \cdot 10^{10} \Pi \mathrm{a} ;$ $v_{1}^{1}=v_{1}^{2}=0.3 ; \quad \rho_{1}=\rho_{2}=\rho_{j}=2.7 \cdot 10^{3} \mathrm{\kappa} \Gamma / \mathrm{M}^{3}$.

Нормальная импульсная нагрузка задавалась в виде

$$
P_{3}=A \cdot \sin \frac{\pi t}{T}[\eta(t)-\eta(t-T)],
$$

где $A$ - амплитуда нагрузки; $T$ - длительность нагрузки. В рассчетах полагалось $A=10^{6}$ Па; $T=50 \cdot 10^{-6}$ с. Подкрепляющие элементы расположены в точках $x_{j}=[11+(k-1) \cdot 15] \cdot \Delta x, k=1 \div 5, \Delta x=L / 80$.

Полученные численные результаты дают возможность характеризовать напряженно - деформируемое состояние трехслойной упругой 
структуры цилиндрического типа в любой момент времени на исследуемом временном интервале согласно вышеуказанных постановок. Расчеты проводились на временном интервале $0 \leq t \leq 40 T$. В частности, на рис. 4.1 и рис. 4.2 приведены зависимости величин прогиба $u_{3}$ от пространственной координаты $x$ в моменты времени $t=6 T$ и $t=7 T$ соответственно (в эти моменты величина $u_{3}$ достигает максимального значения на исследуемом временном интервале $t$ ). Кривая с индексом 1 соответствует теории с дискретным размещением ребер (внутренний слой), с индексом 2 - (внешний слой), с индексом 3 - конструктивно-ортотропной теории трехслойных оболочек с заполнителем. Согласно приведенных численных данных наблюдается качественное и количественное различие в полученных результатах. Учет дискретности размещения ребер (на рисунках это точки соединения кривых с индексом 1 и 2) приводит к более густому волнообразованию величины $u_{3}$ по длине конструкции. Расчеты по конструктивно-ортотропной модели дают некоторые интегральные кривые, которые находятся в пределах изменения величины $u_{3}$ внутреннего и внешнего слоев согласно теории с учетом дискретности ребер. Как предельный случай, в момент времени $t=7 T$ величина $u_{3}$ (кривая с индексом 3) огибает соответствующие кривые (с индексами 1 и 2).

Анализируя количественный характер приведенных результатов видно, что различия по максимальным величинам $u_{3}$ согласно приведенных теорий достигает $40 \%$.

Рассматривалась задача определения напряженно-деформированного состояния цилиндрической оболочки с дискретным заполнителем при продольной краевой нагрузке. Полагалось, что к свободному краю при $x=0$ прикладывается импульсная продольная нагрузка, а другой конец оболочки жестко защемлен. Граничные условия для данной задачи при $x=0$ имеют вид

$$
T_{11}^{k}=\frac{1}{2} F(t), \bar{T}_{13}^{k}=0, \quad M_{11}^{k}=0, \quad k=1,2 .
$$

Для случая жесткого защемления при $x=L$ полагалось

$$
u_{1}^{k}=u_{3}^{k}=\phi_{1}^{k}=0, \quad k=1,2 .
$$

Нестационарная импульсная нагрузка задавался в виде (4.2). Расчеты проводились при следующих геометрических и физико-механических параметрах: 


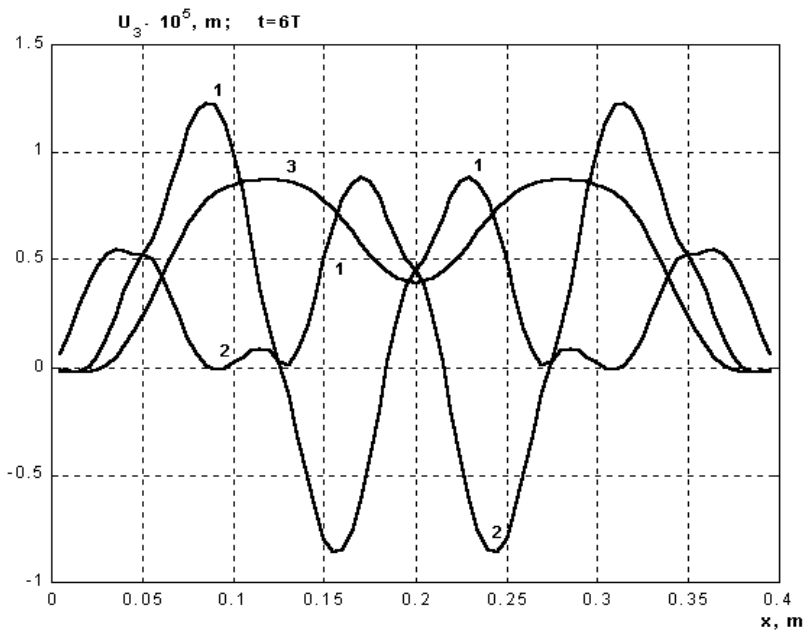

Рис. 4.1. Зависимость величины $u_{3}$ от пространственной координаты $x$ в момент времени $t=6 T$

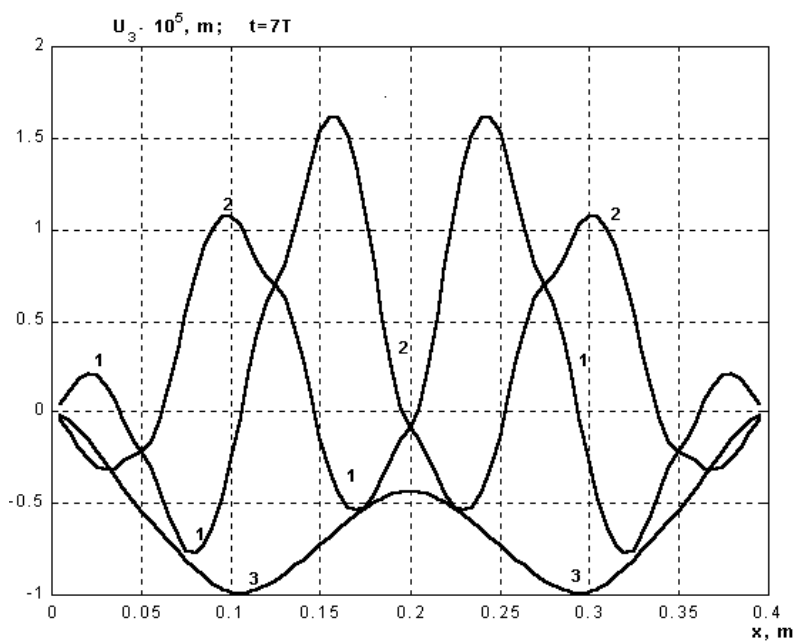

Рис. 4.2. Зависимость величины $u_{3}$ от пространственной координаты $x$ в момент времени $t=7 T$ 


$$
\begin{gathered}
L / h_{1}=40 ; R_{1} / h_{1}=10 ; h_{1}=h_{2} ; H_{j} / h_{1}=2 ; \quad F_{j}=H_{j} h_{1} ; v_{1}^{1}=v_{1}^{2}=0,3 ; \\
E_{1}^{1}=E_{1}^{2}=E_{j}=7 \cdot 10^{10} \Pi \mathrm{\Pi} ; \quad \rho_{1}=\rho_{2}=\rho_{j}=2.7 \cdot 10^{3} \kappa \Gamma / \mathrm{M}^{3} .
\end{gathered}
$$

При решении задач рассматривались следующие случаи дискретного подкрепления $k=1, k=3, k=5, k=7$, где $k$ - количество подкрепляющих ребер, которые равномерно расположены вдоль длины оболочечной структуры. На рис. 4.3 приведены зависимости величины $\sigma_{22}$ от пространственной координаты х в момент времени $t=7 T$, соответствующее достижению максимальных величин $\varepsilon_{11}$. Кривые 1 и 3 соответствуют случаям подкрепления:

$$
k=1(x=L / 2) ; k=3\left(x_{k}=k L / 4, \quad k=\overline{1,3}\right) ; k=5\left(x_{k}=k L / 6, k=\overline{1,5}\right) .
$$

Для случаев $k=1$ и $k=3$ можно визуально определить расположение ребер и их характер влияния на напряженно-деформированное состояние трехслойной структуры. Для случаев $k=5$ и $k=7$ проведено сравнение с результатами расчетов по максимальным величинам $\sigma_{11} \mathrm{i} \sigma_{22}$ согласно конструктивно-ортотропной модели трехслойных оболочек. Учет дискретности заполнителя приводит к увеличению значений $\sigma_{11} \mathrm{i} \sigma_{22}$ (по сравнению с результатами по конструктивно-ортотропной модели трехслойных оболочек) в области жесткого защемления на 15-20\%.

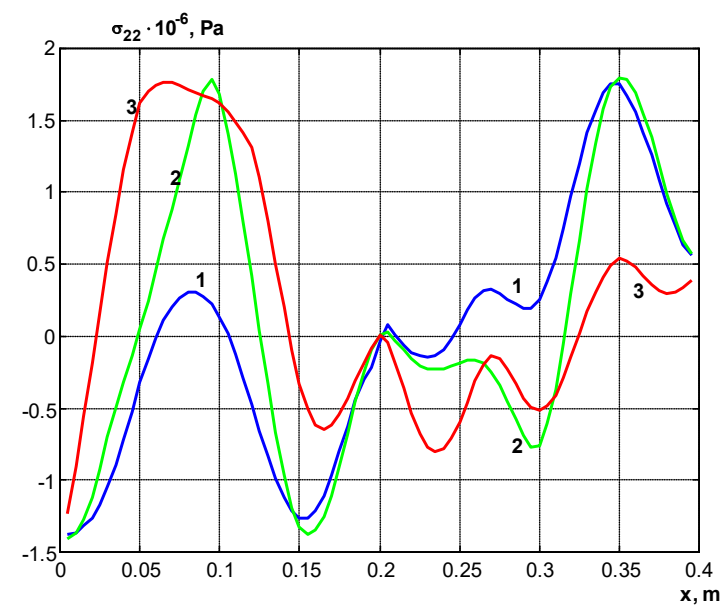

Рис. 4.3. Зависимость величины $\sigma_{22}$ от пространственной координаты $x$ в момент времени $t=7 T$ 
Сферическая оболочка. Дискретно-неоднородна по толщине упругая структура сферического типа представляет собой систему, состоящую из внутренней и внешней гладких сферических оболочек (внутренней и внешней обшивки) с соответствующими толщинами и радиусами срединных поверхностей. Оболочки жестко соединены между собой системой ребер. Полагается, что на трехслойную конструкцию приложено действие внутреннего (или внешнего) нестационарном распределенного нормальной нагрузки $P_{3}(s, t)$, де $s, t-$ пространственная и временная координаты.

Уравнение колебаний трехслойной оболочки сферического типа с внутренним ребристым заполнителем имеют следующий вид при

$$
A_{1}^{k}=R^{k}, \quad A_{2}^{k}=R^{k} \sin \alpha_{1}
$$

- в гладкой области

$$
\begin{gathered}
\frac{1}{\sin \alpha_{1}} \frac{\partial}{\partial s_{k}}\left(\sin \alpha_{1} T_{11}^{k}\right)-\frac{\operatorname{ctg} \alpha_{1}}{R^{k}} T_{22}^{k}+\frac{\bar{T}_{13}^{k}}{R^{k}}=\rho_{k} h_{k} \frac{\partial^{2} u_{1}^{k}}{\partial t^{2}}, \\
\frac{1}{\sin \alpha_{1}} \frac{\partial}{\partial s_{k}}\left(\sin \alpha_{1} \bar{T}_{13}^{k}\right)-\frac{T_{11}^{k}}{R^{k}}-\frac{T_{22}^{k}}{R^{k}}+P_{3}=\rho_{k} h_{k} \frac{\partial^{2} u_{3}^{k}}{\partial t^{2}}, \\
\frac{1}{\sin \alpha_{1}} \frac{\partial}{\partial s_{k}}\left(\sin \alpha_{1} M_{11}^{k}\right)-\frac{\operatorname{ctg} \alpha_{1}}{R^{k}} M_{22}^{k}-T_{13}^{k}=\frac{\rho_{k} h_{k}^{3}}{12} \frac{\partial^{2} \phi_{1}^{k}}{\partial t^{2}}, \quad k=1,2 ;
\end{gathered}
$$

- на линии разрыва $\alpha_{1}=\alpha_{1 j}$

$$
\begin{gathered}
\sum_{i=1}^{2} T_{11}^{k \pm}=\rho_{j} F_{j} \frac{\partial^{2} u_{1 j}}{\partial t^{2}}, \quad \sum_{i=1}^{2} \bar{T}_{13}^{k \pm}=\rho_{j} F_{j} \frac{\partial^{2} u_{3 j}}{\partial t^{2}}, \\
\sum_{i=1}^{2}\left(M_{11}^{k \pm} \mp h_{j} T_{11}^{k \pm}\right)=\rho_{j} I_{k r j} \frac{\partial^{2} \phi_{1 j}}{\partial t^{2}}, \quad k=1,2 ; \quad j=\overline{1, J} .
\end{gathered}
$$

Уравнения колебаний (4.3), (4.4) дополняются соответствующими граничными и начальными условиями.

Как числовой пример рассматривалась задача динамического поведения трехслойной полусферической оболочки при внутренней распределенной импульсной нагрузке $P_{3}(s, t)$. Покладалось, что один край оболочки при $\alpha_{1}=\alpha_{10}$ свободный, а другой край оболочки при $\alpha_{1}=\alpha_{1 N}$ жестко защемлен.

Граничные условия для свободного края при $\alpha_{1}=\alpha_{10}$ имеют следующий вид 


$$
T_{11}^{k}=0, \bar{T}_{13}^{k}=0, \quad M_{11}^{k}=0, \quad k=1,2 .
$$

Для случая жесткого защемления при $\alpha_{1}=\alpha_{1 N}$ полагалось $u_{1}^{k}=u_{3}^{k}=\phi_{1}^{k}=0, \quad k=1,2$.

Нестационарная импульсная нагрузка задавалась в виде (4.2). Рассчеты проводились при следующих геометрических и физико-механических параметрах:

$$
E_{1}^{1}=E_{1}^{2}=E_{j}=7 \cdot 10^{10} \Pi \mathrm{a} ; v_{1}^{1}=v_{1}^{2}=0,3 ; \rho_{1}=\rho_{2}=\rho_{j}=2.7 \cdot 10^{3} \mathrm{\kappa} \Gamma / \mathrm{M}^{3},
$$

$R_{1}=0,3 \mathrm{~m} ; R_{1} / h_{1}=30 ; h_{1}=h_{2} ; h_{j}=2 h ; \quad F_{j}=2 \cdot 10^{-4} \mathrm{M}^{2} ; \alpha_{10}=\pi / 12 ; \alpha_{1 N}=\pi / 2$. Дискретные подкрепляющие элементы расположены в точках

$$
\alpha_{j}=[3+(m-1) \cdot 16] \cdot \Delta \alpha, m=\overline{1,5}, \Delta \alpha=\left(\alpha_{1 N}-\alpha_{10}\right) / 80 .
$$

Полученные численные результаты позволяют проводить анализ напряженно-деформированного состояния трехслойной упругой структуры сферического типа в произвольный момент времени (расчеты проводились на временном интервале $0 \leq t \leq 40 T$ ) согласно приведенной постановки задачи. В частности, на рисунках 4.5 и 4.6 приведены зависимости величин $u_{3}$ ии $\sigma_{22}$ от пространственной координаты $\alpha_{1}$. На рис. 4.4 кривая с индексом 1 соответствует величине $u_{3}$ внутренней сферической оболочки, кривая с индексом 2 соответствует величине $u_{3}$ внешней сферической оболочки в момент времени $t=7 T$. Точки соединения кривых 1 и 2 указывают на розмещение дискретных ребер.

На рис. 4.5 приведены зависимости величины $\sigma_{22}$ от пространственной координаты в момент времени $t=10 T$. Из представленного графического материала отслеживается влияние дискретного заполнителя на напряженно-деформированное состояние трехслойной структуры сферического типа.

Также рассматривалась задача динамично поведения трехслойной полусферической оболочки (случай, когда обшивки изготовлены из ортотропной материала) с отверстием при внутреннем нестационарном нагружении. Полагалось, что граничные и начальные условия соответствуют предыдущему случаю, то есть граничные условия для свободного края при $\alpha_{1}=\alpha_{10}$ имеют следующий вид

$$
T_{11}^{k}=0, \quad \bar{T}_{13}^{k}=0, \quad M_{11}^{k}=0, \quad k=1,2 ;
$$

а для случая жесткого защемления при $\alpha_{1}=\alpha_{1 N}$ полагалось $u_{1}^{k}=u_{3}^{k}=\phi_{1}^{k}=0, \quad k=1,2$. 


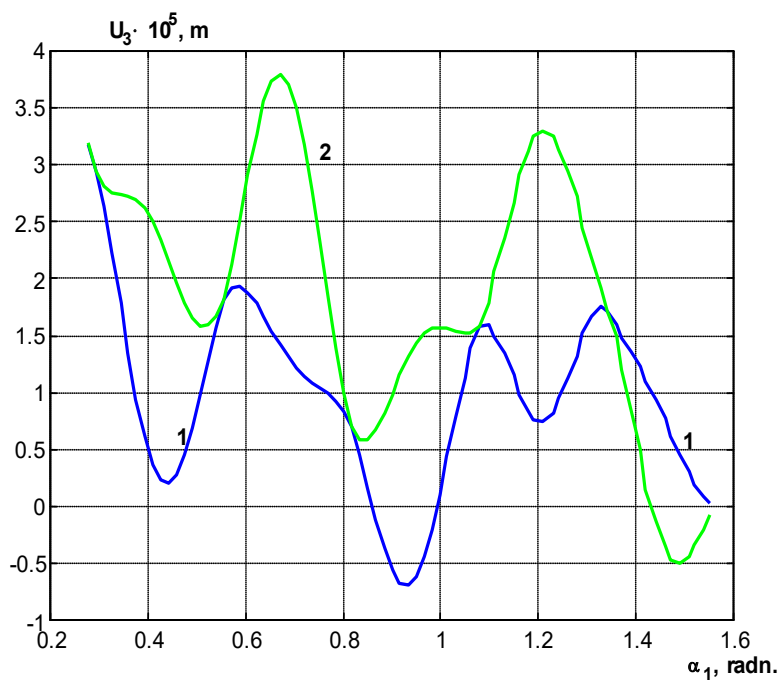

Рис. 4.4. Зависимость величины $u_{3}$ от пространственной координаты $\alpha_{1}$ в момент времени $t=7 T$

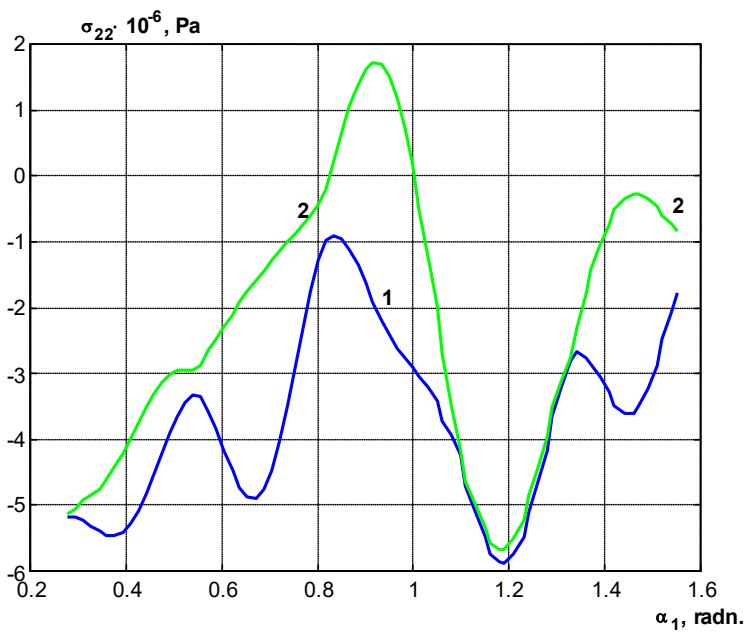

Рис. 4.5. Зависимость величины $\sigma_{22}$ от пространственной координаты $\alpha_{1}$ в момент времени $t=10 T$ 
Внутренняя распределенная нагрузка задавалась в виде

$$
P_{3}=A \cdot[\eta(t)-\eta(t-T)], \text { где } A=2 / \pi \cdot 10^{6} \Pi \mathrm{a} ; T=50 \cdot 10^{-6} \mathrm{c} .
$$

Расчеты проводились при следующих геометрических и физико-механических параметрах:

$$
\begin{gathered}
\alpha_{10}=\pi / 12 ; \alpha_{1 N}=\pi / 2 . E_{1}^{1}=E_{1}^{2}=19 \cdot 10^{9} \Pi \mathrm{a} ; R_{1}=0,3 \mathrm{M} ; \quad v_{1}^{1}=v_{1}^{2}=0,12 ; \\
E_{2}^{1}=E_{2}^{2}=E_{j}=33 \cdot 10^{9} \text { Па; } G_{13}^{1}=G_{13}^{2}=3,69 \cdot 10^{9} ; \rho_{1}=\rho_{2}=\rho_{j}=1,9 \cdot 10^{3} \mathrm{\kappa} \Gamma / \mathrm{M}^{3}, \\
R_{1} / h_{1}=30 ; h_{1}=h_{2} ; h_{j}=2 h ; \quad F_{j}=2 \cdot 10^{-4} \mathcal{M}^{2} .
\end{gathered}
$$

Дискретные подкрепляющие элементы расположены в точках

$$
\alpha_{j}=[3+(m-1) \cdot 16] \cdot \Delta \alpha, m=\overline{1,5}, \Delta \alpha=\left(\alpha_{1 N}-\alpha_{10}\right) / 80 .
$$

Полученные численные результаты позволяют проводить анализ напряженно-деформированного состояния трехслойной упругой структуры сферического типа в произвольный момент времени (расчеты также проводились на временном интервале $0 \leq t \leq 40 T$ ) согласно приведенной постановки задачи. В частности, на рисунке 4.7 приведены зависимости величины $u_{3}$ от пространственной координаты $\alpha_{1}$.

На рис. 4.6 кривая с индексом 1 соответствует величине $u_{3}$ внутренней сферической оболочки, кривая с индексом 2 соответствует величине $u_{3}$ внешней сферической оболочки в момент времени $t=7 T$. Точки соединения кривых 1 и 2 указывают, дискретных ребер.

Коническая оболочка. Дискретно-неоднородна по толщине упругая структура конического типа представляет собой систему, состоящую из внутренней и внешней гладких конических оболочек (внутренняя и внешняя обшивки) с соответствующими толщинами и радиусами. Полагается, что срединные линии этих оболочек параллельные, то есть угол конусности $\alpha$ общий. Оболочки жестко соединены между собой дискретными ребрами. Считается, что трехслойная коническая конструкция нагружена внутренней распределенной нестационарной нормальной нагрузкой $P_{3}(s, t)$, где $s$ и $t$-пространственная и временная координаты.

При рассмотрении осесимметричных колебаний конических оболочек обычно используется система координат $s, t$, причем координата $s$ отсчитывается от вершины конуса. В некоторых случаях, в частности для срезанных конических оболочек, более удобным является использование координаты $s_{1}$, где координата $s_{1}$ отсчитывается от края оболочки. 


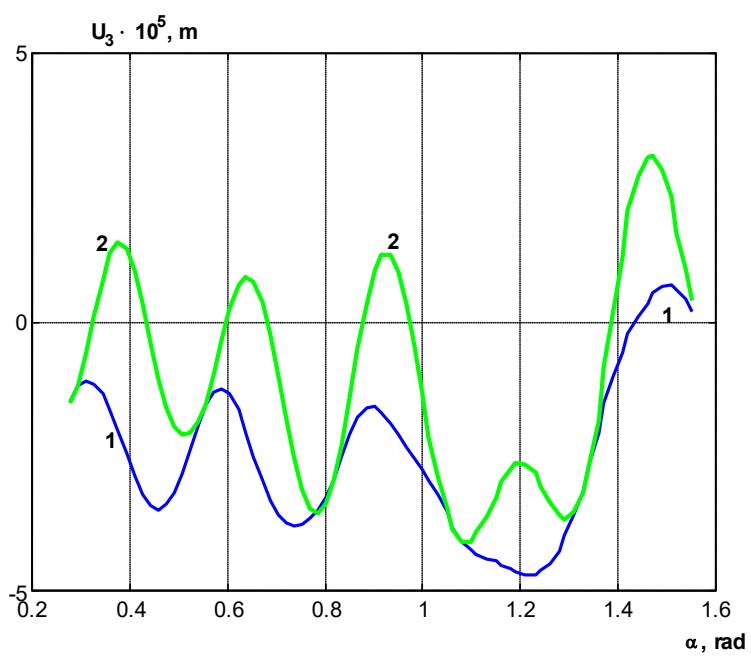

\section{Рис. 4.6. Зависимость величины $u_{3}$ от пространственной} координаты $\alpha_{1}$ в момент времени $t=7 T$

Коэффициенты первой квадратичной формы и кривизны координатной поверхности записываются следующим образом:

$$
A_{1}=1, \quad A_{2}=R_{s}, k_{1}=0, \quad k_{2}=\left(R_{s} \cos \alpha\right)^{-1},
$$

где $\alpha$-кут конусности; $s_{1}$ - текущая координата; $R_{s}=R_{0}+s_{1} \sin \alpha$.

Исходя из приведенных зависимостей и уравнений, запишем уравнение колебаний трехслойной конической оболочки с дискретным заполнителем в следующем виде:

- в гладкой области

$$
\begin{gathered}
\frac{1}{R_{k}} \frac{\partial}{\partial s_{k}}\left(R_{k} T_{11}^{k}\right)-\frac{\sin \alpha}{R^{k}} T_{22}^{k}=\rho_{k} h_{k} \frac{\partial^{2} u_{1}^{k}}{\partial t^{2}}, \\
\frac{1}{R_{k}} \frac{\partial}{\partial s_{k}}\left(R_{k} \bar{T}_{13}^{k}\right)-\frac{\cos \alpha}{R_{k}} T_{22}^{k}+P_{3}(s, t)=\rho_{k} h_{k} \frac{\partial^{2} u_{3}^{k}}{\partial t^{2}}, \\
\frac{1}{R_{k}} \frac{\partial}{\partial s_{k}}\left(R_{k} M_{11}^{k}\right)-\frac{\sin \alpha}{R_{k}} M_{22}^{k}-T_{13}^{k}=\frac{\rho_{k} h_{k}^{3}}{12} \frac{\partial^{2} \phi_{1}^{k}}{\partial t^{2}}, \quad k=1,2 .
\end{gathered}
$$

На линиях разрывов уравнение колебаний записываются в виде 


$$
\begin{gathered}
\sum_{i=1}^{2} T_{11}^{i \pm}=\rho_{j} F_{j} \frac{\partial^{2} u_{1 j}}{\partial t^{2}}, \quad \sum_{i=1}^{2} \bar{T}_{13}^{i \pm}=\rho_{j} F_{j} \frac{\partial^{2} u_{3 j}}{\partial t^{2}}, \\
\sum_{i=1}^{2}\left(M_{11}^{i \pm} \mp h_{j} T_{11}^{i \pm}\right)=\rho_{j} I_{\kappa p j} \frac{\partial^{2} \phi_{1 j}}{\partial t^{2}} .
\end{gathered}
$$

В уравнениях (4.5) величины $T_{11}^{i \pm}, \bar{T}_{13}^{i \pm}=T_{11}^{i \pm}+\theta_{11}^{i \pm}, M_{11}^{i \pm}(i=1,2)$ соответствуют усилиям-моментам, которые действуют на $j$ - й дискретный элемент $s_{i}=s_{i j}$. Уравнение колебаний (4.5), (4.6) дополняются соответствующими граничными и начальными условиями.

Численный алгоритм решения вышеуказанных задач основывается на использовании алгоритма, который изложен в третьей главе данной работы.

Рассматривалась задача динамического деформирования трехслойной конической оболочки с жестко ущемленными торцами под действием внутренней распределенной нагрузки $P_{3}(s, t)$. Граничные условия при $s=s_{0}, \quad s=s_{N}$ имеют вид: $u_{1}^{k}=u_{3}^{k}=\phi_{1}^{k}=0, \quad k=1,2$.

Нестационарное импульсная нагрузка задавался в виде (4.2).

Расчеты проводились при следующих геометрических и физико-механических параметрах:

$$
\begin{gathered}
\alpha=\pi / 12 ; E_{1}^{1}=E_{1}^{2}=E_{j}=7 \cdot 10^{10} \Pi \mathrm{a} ; \rho_{1}=\rho_{2}=\rho_{j}=2.7 \cdot 10^{3} \mathrm{\kappa} / \mathrm{M}^{3}, \\
v_{1}^{1}=v_{1}^{2}=0,3 ; R_{1}=0,3 \mathrm{M} ; R_{1} / h_{1}=30 ; h_{1}=h_{2} ; h_{j}=2 h ; F_{j}=2 \cdot 10^{-4} \mathrm{M}^{2} .
\end{gathered}
$$

Дискретные подкрепляя элементы расположены в точках

$$
s_{j}=[3+(k-1) \cdot 16] \cdot \Delta s, k=\overline{1,5}, \Delta s=\left(s_{N}-s_{0}\right) / 80 .
$$

Полученные численные результаты позволяют проводить анализ напряженно-деформированного состояния трехслойной упругой структуры конического типа в любой момент времени (расчеты проводились при $0 \leq t \leq 40 T$ ). В частности, на рис. 4.7 приведены зависимости величин $u_{3}$ от пространственной координаты $s$. Кривая с индексом 1 соответствует величине $u_{3}$ Кривая с индексом 1 соответствует величине $u_{3}$ внешней конической оболочки в момент времени $t=4 T$ (Время достижения максимального значения величины $u_{3}$ ). Точки соединения кривых 1 и 2 указывают на расположение дискретных ребер. Исходя из представленного графического материала можно визуально определить влияние конусности структуры на антисимметричности 


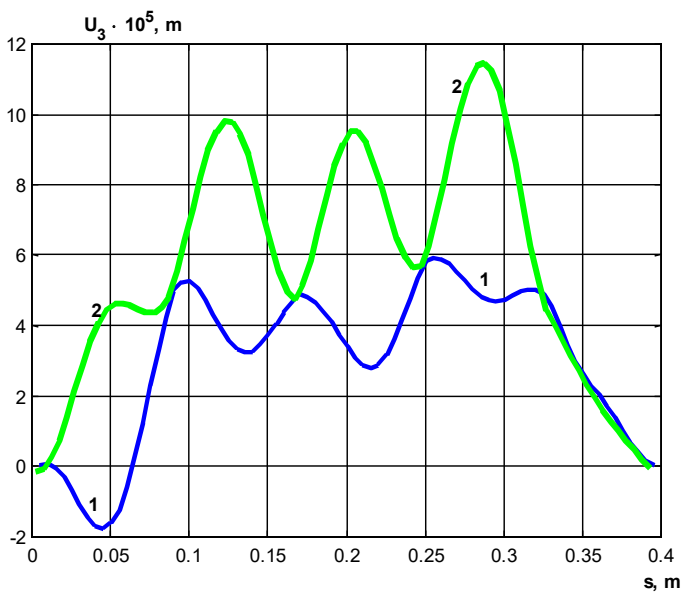

Рис. 4.7 Зависимость величины $u_{3}$ от пространственной координаты S в момент времени $t=4 T$

распределения величины по пространственной координате (как частный случай, для цилиндрической оболочки в случае $\alpha=0$ наблюдается симметричная картина относительно оси s).

\section{5. Достоверность полученных численных результатов}

Достоверность полученных в работе результатов определяется корректностью постановок задач; теоретическим обоснованием используемых конечно-разностных схем; контролируемой точностью численных расчетов; соответствием установленных закономерностей общим свойствам колебаний тонкостенных элементов конструкций.

Корректность постановки задач достигается использованием известных уравнений теории оболочек и стержней типа Тимошенко, аппроксимирующих исходные уравнения трехмерной теории упругости. При выводе уравнений получены уравнения колебаний обшивок в гладкой области, которые представляют собой, в общем случае, оболочки вращения, и уравнения колебаний подкрепляющих ребристых элементов (продольные и поперечные ребра). Нетрудно показать, что указанные уравнения по классификации уравнений в частных произ- 
водных являются уравнениями гиперболического типа, которые являются аппроксимацией колебательных уравнений трехмерных упругих тел и достаточно корректно [3; 5] воспроизводят волновые процессы в неоднородных оболочечных структурах с учетом пространственных разрывов.

Численные алгоритмы приближенных решений исходных уравнений основываются на использовании интегро-интерполяционного метода построения разностных схем. При построении разностных схем кинематические величины соотносятся к разностным точкам с целыми индексами, а величины деформаций и усилий-моментам соотносятся к разностным точкам с полуцелыми индексами. Как показано в работе [5] такая аппроксимация исходных кинематических и статических величин позволяет выполнять закон сохранения полной механической энергии упругой структуры на разностном уровне. Численный алгоритм базируется на использовании отдельных конечно-разностных соотношений в гладкой области и на линиях пространственных разрывов со вторым порядком точности по пространственным и временной координатам. При этом, в ряде случаев проведено теоретическое исследование вопросов устойчивости разностных уравнений. В частности, для конечно-разностных уравнений колебаний трехслойных цилиндрических оболочек с учетом дискретности заполнителя при осесимметричных и не осесимметричных колебаниях [2]. Исходная аппроксимация конечно-разностных уравнений (второй порядок точности по пространственным и временной координатам) и условие устойчивости разностных уравнений, согласно теории разностных схем $[1 ; 4]$ позволяют говорить о сходимости численных результатов, полученных с использованием указанных конечно-разностных схем.

При конкретных численных расчетах достигалась практическая сходимость результатов, как по пространственным координатам так и по временной координате. В качестве примера рассмотрим достижение практической сходимости в задаче о вынужденных осесимметричных нестационарных колебаний трехслойной цилиндрической оболочки при воздействии на нее внутренней распределенной нагрузки. Полагалось, что края оболочки жестко защемлены. Рассматривался случай дискретного подкрепления тремя кольцевыми ребрами в точках $x_{j}=j L / 4, j=\overline{1,3}$. Результаты расчетов приведены в следующих 
таблицах, в колонках которых приведены максимальные величины $u_{3}$ и $\sigma_{22}$ по пространственной и временной координатам.

Таблица 4.1

Числеленная сходимость величин $u_{3}$ и $\sigma_{22}$ по пространственной координате

\begin{tabular}{|c|c|c|c|c|}
\hline & $\frac{u_{3} \cdot 10^{-5}, M}{x=L / 8}$ & $\frac{u_{3} \cdot 10^{-5}, M}{x=3 L / 8}$ & $\frac{\sigma_{22} \cdot 10^{6}, \Pi a}{x=L / 8}$ & $\frac{\sigma_{22} \cdot 10^{6}, \Pi a}{x=3 L / 8}$ \\
\hline $\mathrm{N}=80$ & 0.123 & 0.108 & 0.975 & -0.929 \\
\hline $\mathrm{N}=160$ & 0.174 & 0.168 & 1.300 & -1.220 \\
\hline $\mathrm{N}=320$ & 0.174 & 0.168 & 1.302 & -1.21 \\
\hline
\end{tabular}

Таблиця 4.2

Числеленная сходимость величин $u_{3}$ и $\sigma_{22}$ по пространственной координате

\begin{tabular}{|c|c|c|c|c|}
\hline & $\frac{u_{3} \cdot 10^{-5}, M}{x=L / 8}$ & $\frac{u_{3} \cdot 10^{-5}, M}{x=3 L / 8}$ & $\frac{\sigma_{22} \cdot 10^{6}, \Pi a}{x=L / 8}$ & $\frac{\sigma_{22} \cdot 10^{6}, \Pi a}{x=3 L / 8}$ \\
\hline $\mathrm{K}=0.92$ & 0.174 & 0.168 & 1.300 & -1.220 \\
\hline $\mathrm{K}=0.8$ & 0.174 & 0.168 & 1.300 & -1.220 \\
\hline
\end{tabular}

Таблица 4.1 соответствует результатам численной сходимости величин $u_{3}$ и $\sigma_{22}$ по пространственной координате $\mathrm{x}$ в зависимости от количества интервалов разбиения длины оболочки L для случаев $\mathrm{N}=80, \mathrm{~N}=160$ та $\mathrm{N}=320$. Как видно из приведенных результатов, удовлетворительная точность достигается при $\mathrm{N}=160$. Если принять расчеты при $\mathrm{N}=320$ за точные, то относительная погрешность расчетов при $\mathrm{N}=160$ по величинам $\sigma_{22}$ в точках $x=L / 8$ и $x=3 L / 8$ меньше $1 \%$. Таблица 4.2 соответствует результатам численной сходимости величин $u_{3}$ и $\sigma_{22}$ по временной координате $\mathrm{t}$ в зависимости от числа Куранта К для случая $\mathrm{N}=160$, где число К удовлетворяет равенству

$$
\Delta t=K \cdot \Delta x / c_{11},
$$

где $c_{11}=E_{1} /\left(\rho-v_{1} v_{2}\right)$.Последние две строки таблицы показывают на практическую сходимость по временной координате $t$ при $\mathrm{K}=0,92$ и $\mathrm{K}=0,8$. 
В частности, результаты представленные в таблице 4.2 свидетельствуют об эффективности численных методов, используемых в данной работе - расчеты проводились при числах Куранта близких к $1(K=0,92)$.

\section{6. Выводы}

Данная работа посвящена исследованию нестационарных осесимметричных колебаний трехслойных оболочек вращения с учетом дискретности заполнителя. На основе вариационного принципа Гамильтона-Остроградского получены уравнения колебаний и естественные граничные условия для трехслойных оболочек вращения с учетом дискретности заполнителя в рамках гипотез теории оболочек и стержней типа Тимошенко. Развит эффективный численный метод решения динамических задач трехслойных оболочек вращения. В основе численного алгоритма лежит явная конечно-разностная схема интегрирования типа "крест" по временной и пространственным координатам. В частном случае, для трехслойных цилиндрических оболочек проведено теоретическое исследование условий устойчивости явных по временной координате конечно - разностных схем. Решены задачи осесимметричных колебаний трехслойных оболочек вращения (цилиндрических, сферических, конических) в широком диапазоне изменения геометрических и физико-механических параметров при разных видах граничных условий и типах нагружения. Проведен сравнительный анализ результатов исследования динамического поведения трехслойных цилиндрических оболочек в рамках теории неоднородных оболочек с учетом дискретности наполнителя и конструктивно ортотропной теории трехслойных оболочек с наполнителем. Исследовано численную сходимость полученных результатов на примере динамического поведения жестко защемленной по краям трехслойной цилиндрической оболочки с учетом дискретного ребристого наполнителя при распределенном нагружении. Полученные результаты позволяют анализировать особенности динамического деформирования трехслойных оболочек вращения с учетом дискретности наполнителя.

В дальнейшем, перспективным есть постановки и численные решения двумерных по пространственным координатам задач динамики теории трехслойных оболочек с учетом дискретного заполнителя при нестационарных нагружениях. 


\section{Список литературы:}

1. Годунов С.К., Рябенький В.С. Разностные схемы. Москва : Наука, 1977.

2. Головко К.Г., Луговой П.З., Мейш В.Ф. Динамика неоднородных оболочек при нестационарных нагрузках. Киев : Изд.-полиграф. центр «Киевский ун-Т», 2012.

3. Григолюк Э.И., Селезов И.Т. Неклассические теории колебаний стержней пластин и оболочек. Итоги науки и техники. ВИНИТИ. Механика деформ. твердого тела, 1973. 1980.

4. Марчук Г.И. Методы вычислительной математики. Москва : Наука,

5. Навал И.К., Пацюк В.И., Римский В.К. Нестационарные волны в деформируемых средах. Кишенев : Штиница, 1986.

6. Перцев А.К., Платонов Э.Г. Динамика оболочек и пластин (нестационарные задачи). Ленинград : Судостороение, 1987.

7. Самарский А.А. Теория разностных схем. Москва : Наука, 1977.

8. Meish V.F., Meish Yu.A., \& PavlyukA.V. (2018). Dynamics of a Three-Layer Elliptic Cylindrical Shells Reinforced with Discrete Rings. International Applied Mechanics, 54(2): 172-179.

9. Meish V.F., \& Maiborodina N.V. (2018). Stress state of discretely stiffened ellipsoidal shells under a nonstationary normal load. International Applied Mechanics, 54(2): 675-686.

10. Meish V.F., Meish Yu.A., \& Arnauta N.V. (2019). Numerical Analysis of Nonstationary Vibrations of Discretely Reinforced Multilayer Shells of Different Geometry. International Applied Mechanics, 55(4): 426-433.

11. Tornabene F., Fantuzzi N., Bacciocchi M., \& Dimitri R. (2015). Free vibrations of composite oval and elliptic cylinders by the generalized differential quadrature method. Thin Walled Struct. 97: 114-129.

12. Shulga N.A., Meish V.F., \& Khamrenko Yu.A. (1999). Nonstationary Vibrations of Three-layered Cylindrical Shells under Axisymmetric Loading. International Applied Mechanics, 35(8): 754-757.

\section{References:}

1. Ghodunov S.K., Rjabenjkyj V.S. (1977). Raznostnye skhemy. Moskva: Nauka. (in Russian)

2. Gholovko K.Gh., Lughovoj P.Z., Mejsh V.F. (2012). Dynamyka neodnorodnykh obolochek pry nestacyonarnykh naghruzkakh. Kyev: Yzd.-polyghraf. centr «Kyevskyj un-t». (in Russian)

3. Ghrygholjuk E.Y., Selezov Y.T. (1973). Neklassycheskye teoryy kolebanyj sterzhnej plastyn y obolochek. Ytoghy nauky y tekhnyky. VYNYTY. Mekhanyka deform. tverdogho tela. (in Russian)

4. Marchuk Gh.Y. (1980). Metody vychyslyteljnoj matematyky. Moskva: Nauka. (in Russian)

5. Naval Y.K., Pacjuk V.Y., Rymskyj V.K. (1986). Nestacyonarnue volny v deformyruemykh sredakh. Kyshenev: Shtynyca. (in Russian) 
6. Percev A.K., Platonov E.Gh. (1987). Dynamyka obolochek y plastyn (nestacyonarnye zadachy). Lenynghrad: Sudostoroenye. (in Russian)

7. Samarskyj A.A. (1977). Teoryja raznostnykh skhem. Moskva: Nauka. (in Russian)

8. Meish V.F., Meish Yu.A., \& PavlyukA.V. (2018). Dynamics of a Three-Layer Elliptic Cylindrical Shells Reinforced with Discrete Rings. International Applied Mechanics, 54(2): 172-179.

9. Meish V.F., \& Maiborodina N.V. (2018). Stress state of discretely stiffened ellipsoidal shells under a nonstationary normal load. International Applied Mechanics, 54(2): 675-686.

10. Meish V.F., Meish Yu.A., \& Arnauta N.V. (2019). Numerical Analysis of Nonstationary Vibrations of Discretely Reinforced Multilayer Shells of Different Geometry. International Applied Mechanics, 55(4): 426-433.

11. Tornabene F., Fantuzzi N., Bacciocchi M., \& Dimitri R. (2015). Free vibrations of composite oval and elliptic cylinders by the generalized differential quadrature method. Thin Walled Struct. 97: 114-129.

12. Shulga N.A., Meish V.F., \& Khamrenko Yu.A. (1999). Nonstationary Vibrations of Three-layered Cylindrical Shells under Axisymmetric Loading. International Applied Mechanics, 35(8): 754-757. 